\title{
Cyclodextrin inclusion complexes with caffeoylquinic acids as bioactive compounds
}

\author{
$\underline{\text { Silvia Navarro-Orcajada }}{ }^{1}$, Adrián Matencio ${ }^{2}$, Francisco García-Carmona ${ }^{1}$ and José Manuel López- \\ Nicolás $^{1 *}$
}

${ }^{1}$ Departamento de Bioquímica y Biología Molecular-A, Facultad de Biología, Universidad de Murcia - Regional
Campus of International Excellence "Campus Mare Nostrum, E-30100 Murcia, Spain
${ }^{2}$ Dipartimento Di Chimica, Università di Torino, via P. Giuria 7, 10125 Torino, Italy
* Email: josemln@ @um.es / Tlf.: +34 868884786

SESSION: B. Cyclodextrins in Pharmaceutics

\begin{abstract}
Neochlorogenic acid, a less-studied caffeoylquinic acid, isomer of chlorogenic acid, has been seen to possess antioxidant, antifungal, anti-inflammatory and anticarcinogenic effects, which makes it an interesting bioactive compound for incorporation in drugs, nutraceuticals or functional foods. However, its poor solubility in water and susceptibility to oxidation make such a task difficult. To overcome that, its encapsulation in cyclodextrins (CDs) is proposed. The fluorescence of neochlorogenic acid in different $\mathrm{pH}$ conditions was analyzed, and caffeic acid was proved to be the fluorescent moiety in the molecule. An encapsulation model whereby the ligand poses two potential complexation sites (caffeic and D-(-)-quinic moieties), showed that $\alpha$-CD and HP- $\beta$-CD formed the best inclusion complexes with neochlorogenic acid, followed by $\mathrm{M}-\beta-\mathrm{CD}, \beta-\mathrm{CD}$ and $\gamma-\mathrm{CD}$. Molecular docking with the two best $\mathrm{CDs}$ gave better scores for $\alpha-\mathrm{CD}$, despite HP- $\beta-\mathrm{CD}$ providing stabilization through H-bonds. The encapsulation of chlorogenic acid led to a similar $\mathrm{CD}$ order and scores, although constants were higher for $\alpha-C D, \beta-C D$ and $\mathrm{M}-\beta-\mathrm{CD}$, lower for HP- $\beta-\mathrm{CD}$, and negligible for $\gamma$-CD. The solubility and the susceptibility to oxidation of neochlorogenic acid improved after complexation with $\alpha-\mathrm{CD}$ and HP- $\beta-\mathrm{CD}$, while the antioxidant activity of both isomers was maintained. These results could lead to obtaining more stable inclusion complexes with caffeoylquinic acids for applications in the pharmaceutical industry.
\end{abstract}

KEYWORDS: Neochlorogenic acid; Caffeoylquinic acids; Cyclodextrins; Encapsulation; Fluorescence

ACKNOWLEDGEMENTS: This research was funded by the Spanish Ministry of Science and Innovation, project AGL2017-86526-P (MCI/AEI/FEDER, UE) and by the "Programa de Ayudas a Grupos de Excelencia de la Región de Murcia, Fundación Séneca, Agencia de Ciencia y Tecnología de la Región de Murcia (Spain)" (Project 19893/GERM/15). This work is the result of a predoctoral contract for the training of research staff (Silvia Navarro-Orcajada) and also an aid to postdoctoral training and improvement abroad (for Adrián Matencio) both financed by the Consejería de Empleo, Universidades, Empresa y Medio Ambiente of the CARM, through the Fundación Séneca-Agencia de Ciencia y Tecnología de la Región de Murcia. 